\title{
Diagnostic delay of pulmonary tuberculosis in patients with acute respiratory distress syndrome associated with aspiration pneumonia: Two case reports and a mini-review from Japan
}

\author{
MAKOTO NAKAO, KAZUKI SONE, YUSUKE KAGAWA, RYOTA KUROKAWA, \\ HIDEFUMI SATO, TAKEFUMI KUNIEDA and HIDEKI MURAMATSU
}

\begin{abstract}
Department of Respiratory Medicine, Kainan Hospital, Aichi Prefectural Welfare Federation of Agricultural Cooperatives, Yatomi, Aichi 498-8502, Japan
\end{abstract}

Received May 18, 2015; Accepted January 8, 2016

DOI: 10.3892/etm.2016.3385

\begin{abstract}
Diagnosing active tuberculosis in elderly patients presents problems due to nonspecific symptoms and complications such as aspiration pneumonia. The current study presents two cases of pulmonary tuberculosis with bilateral pulmonary infiltrates associated with aspiration pneumonia. The two elderly patients developed acute respiratory distress syndrome as a result of aspiration pneumonia. The diagnoses of pulmonary tuberculosis were delayed in both cases, as the patients were diagnosed with active tuberculosis following discharge from hospital. The sputum test for acid-fast bacillus at the time of administration was smear-negative/culture-positive in these patients. They were treated with isoniazid, rifampicin and ethambutol, and nosocomial transmission of tuberculosis from these patients was not reported. The number of elderly patients with aspiration pneumonia is predicted to increase rapidly, and aspiration pneumonia combined with pulmonary tuberculosis is a major medical and healthcare concern in Japan. The present study concludes that physicians should always consider the complication of pulmonary tuberculosis when treating pneumonia patients, in particular in treating elderly patients with pulmonary infiltrates.
\end{abstract}

\section{Introduction}

In Japan, the number of deaths associated with pneumonia is increasing, and pneumonia is now the third leading cause of mortality worldwide, causing $\sim 10 \%$ of all mortalities (1-3). Patients aged $\geq 65$ years account for $>95 \%$ of

Correspondence to: Dr Makoto Nakao, Department of Respiratory Medicine, Kainan Hospital, Aichi Prefectural Welfare Federation of Agricultural Cooperatives, 396 Minamihonden, Maegasucho, Yatomi, Aichi 498-8502, Japan

E-mail: kokoro1979@gmail.com

Key words: tuberculosis, diagnostic delay, aspiration pneumonia, acute respiratory distress syndrome, elderly pneumonia-associated mortalities, and aspiration pneumonia is common in elderly patients (1,3-5). Although the typical symptoms of aspiration pneumonia are fever, coughing and sputum, non-specific symptoms, including appetite loss and impaired consciousness, are also important. The risk of mortality caused by pneumonia increases with age, and the number of elderly patients with aspiration pneumonia is expected to rapidly increase as the human life expectancy increases $(1,4)$.

At present, aspiration pneumonia combined with pulmonary tuberculosis is a major concern in elderly patients (6). Between 1900 and 1950, the prevalence of tuberculosis in Japan was recorded as $>500$ per 100,000 of the population (7). Although the prevalence of tuberculosis was subsequently reduced to 16.7 per 100,000 of the Japanese population in 2012, this figure remains 3-4 times higher than the prevalence of tuberculosis in Europe and North America (8). In Japan, one reason for the relatively high prevalence of tuberculosis is the aging of individuals who were previously infected with the disease (9).

In the present study, the diagnostic delay of pulmonary tuberculosis in two patients with aspiration pneumonia, who developed acute respiratory distress syndrome (ARDS) according to the American-European Consensus Conference (AECC) criteria, is described (10). The patients were diagnosed with active pulmonary tuberculosis following discharge from the hospital. Failure to recognize active pulmonary tuberculosis delays the commencement of anti-tuberculous therapy and may cause nosocomial transmission of pulmonary tuberculosis.

\section{Case report}

The present study was approved by the Ethics Committee of Kainan Hospital, Aichi Prefectural Welfare Federation of Agricultural Cooperatives (Yatomi, Japan).

Case 1. A 92-year-old woman was transported to Kainan Hospital (Yatomi, Japan) by ambulance on September 10th 2013 complaining of vomiting, a high fever and impaired consciousness. The patient had developed aspiration pneumonia 

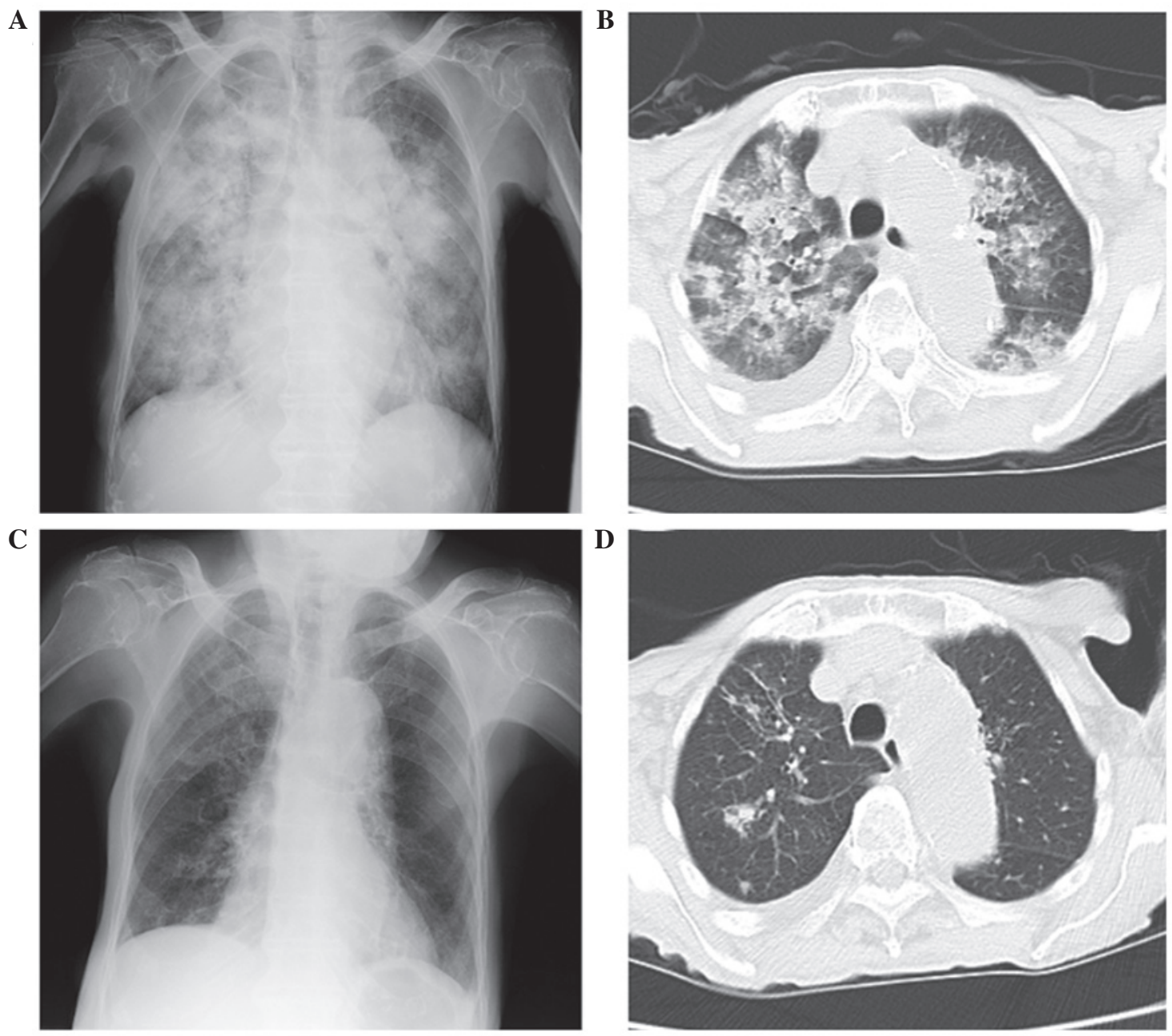

Figure 1. (A) Chest X-ray film presenting diffuse bilateral consolidation. (B) CT scan presenting diffuse bilateral consolidation and right pleural effusion. (C) Chest X-ray film presenting near complete absence of the diffuse bilateral consolidation observed previously, with a remaining pale patchy shadow in the right upper lung field. (D) CT scan presenting nodular opacity with small satellite nodules in the right upper lobe. CT, computerised tomography.

4 months previously, which had successfully been treated with antibiotic therapy $[2 \mathrm{~g}$ ampicillin plus $1 \mathrm{~g}$ sulbactam sodium (Meiji Seika Pharma, Co., Ltd., Tokyo, Japan) every 12 h]. On arrival at our hospital, the patient presented with the following signs: Temperature, $39.2^{\circ} \mathrm{C}$; pulse rate, 103 beats $/ \mathrm{min}$; blood pressure, $92 / 59 \mathrm{mmHg}$; and coarse crackles over the bilateral lungs. Blood test results reported an increase in the percentage of neutrophils [white blood cells: $8,100 / \mathrm{mm}^{3}$ (normal range,

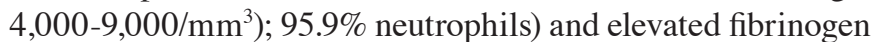
[519 mg/dl (normal range, 200-400 mg/dl)] and C-reactive protein $[7.5 \mathrm{mg} / \mathrm{dl}$ (normal range, $0.0-0.3 \mathrm{mg} / \mathrm{dl}$ )]. In addition, the patient presented with anemia [hemoglobin, $9.2 \mathrm{~g} / \mathrm{dl}$ (normal range, $11.5-14.9 \mathrm{mg} / \mathrm{dl})]$ and low serum albumin $[2.1 \mathrm{~g} / \mathrm{dl}$ (normal range, 4.0-5.0 mg/dl)]. Blood gas analysis recorded a pH of $7.463, \mathrm{CO}_{2}$ partial pressure $\left(\mathrm{PaCO}_{2}\right)$ of $40.8 \mathrm{mmHg}$, $\mathrm{PaO}_{2}$ of $68.4 \mathrm{mmHg}$ and $\mathrm{O}_{2}$ saturation $\left(\mathrm{SaO}_{2}\right)$ of $93.4 \%$. The aforementioned results were determined by oxygen inhalation through a mask with a reservoir bag $(10 \mathrm{l} / \mathrm{min})$. The ratio of $\mathrm{PaO}_{2}$ and fraction of inspired $\mathrm{O}_{2}\left(\mathrm{FiO}_{2}\right)$ was 68.4. Chest X-ray film and computerised tomography (CT) presented diffuse bilateral consolidation and right pleural effusion (Fig. 1A and B). The sputum test for acid-fast bacilli at the time of admission was smear negative. No evidence of left atrial hypertension was detected by ultrasonic cardiography. Therefore, a diagnosis of ARDS due to aspiration pneumonia was clinically established according to the AECC criteria (10).

The patient was treated with $4 \mathrm{~g}$ piperacillin and $0.5 \mathrm{~g}$ tazobactam (both Taiho Pharmaceutical, Co., Ltd., Tokyo, Japan) antibiotics every $8 \mathrm{~h}$ for 10 days, in addition to $\mathrm{O}_{2}$ therapy and intravenous feeding. The treatments proved successful; improved oxygenation and a reduced fever were observed. Therefore, the patient was discharged to a nursing home on September 26th. A chest X-ray film obtained at the time of discharge revealed that the diffuse bilateral consolidation had almost completely disappeared; however, a pale patchy shadow remained in the right upper lung field (Fig. 1C). On October 3rd, an acid-fast bacillus examination of the sputum obtained from the patient upon admission presented with culture-positive results, and a polymerase chain reaction (PCR) analysis for Mycobacterium tuberculosis returned positive. The patient was then readmitted to hospital, and underwent sputum tests for acid-fast bacillus and a chest CT scan. The chest CT scan identified a nodular opacity with small satellite nodules in the right upper lobe; these nodules were suspected to be the focus of the tuberculosis infection (Fig. 1D). Based on the chest CT images and the results of the acid-fast bacillus re-examination 
A

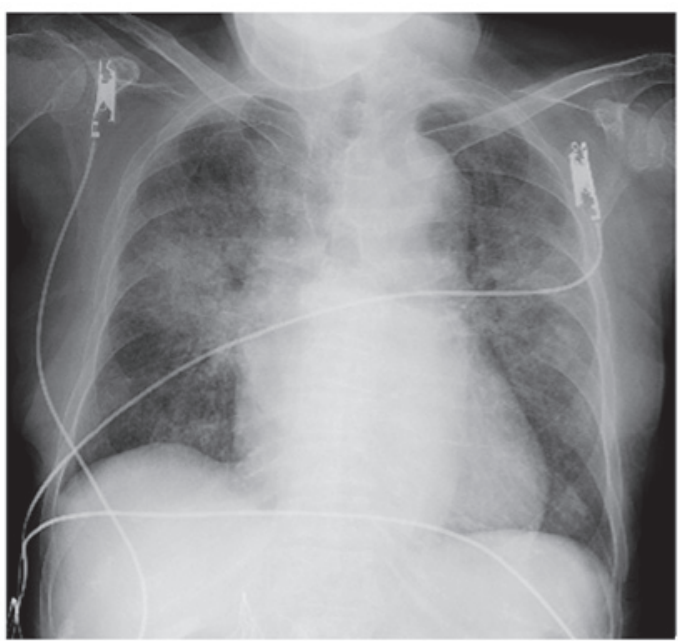

C

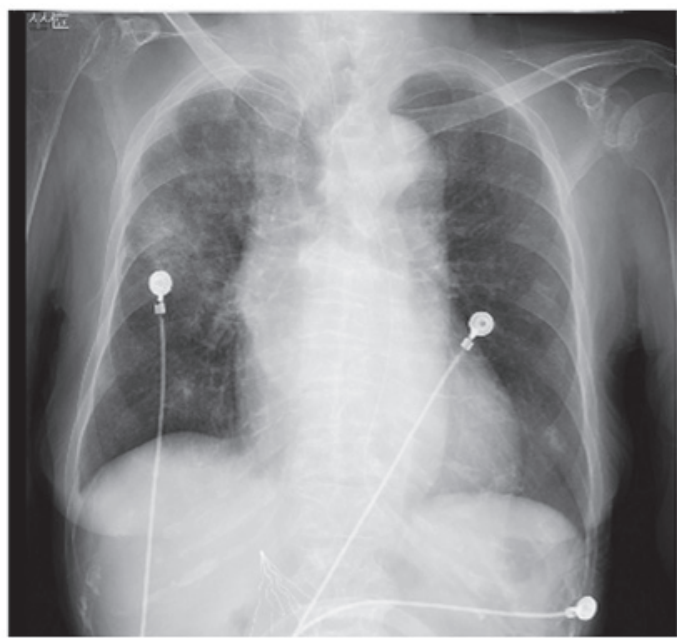

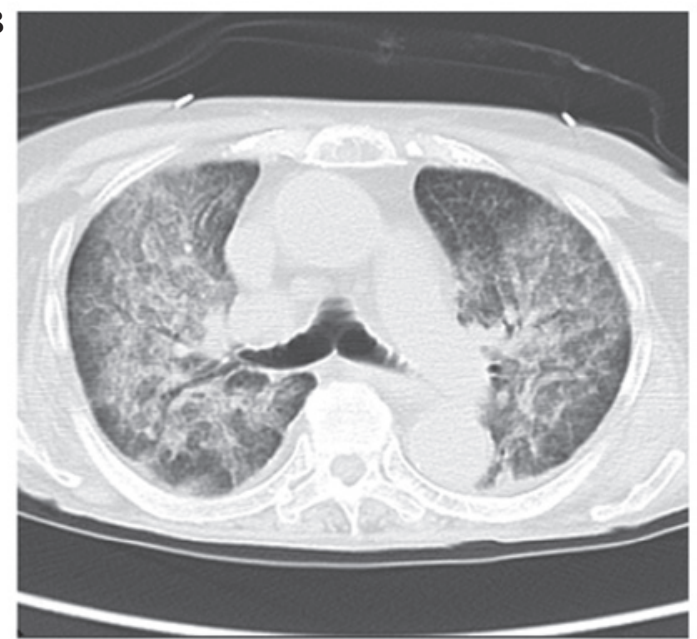

D

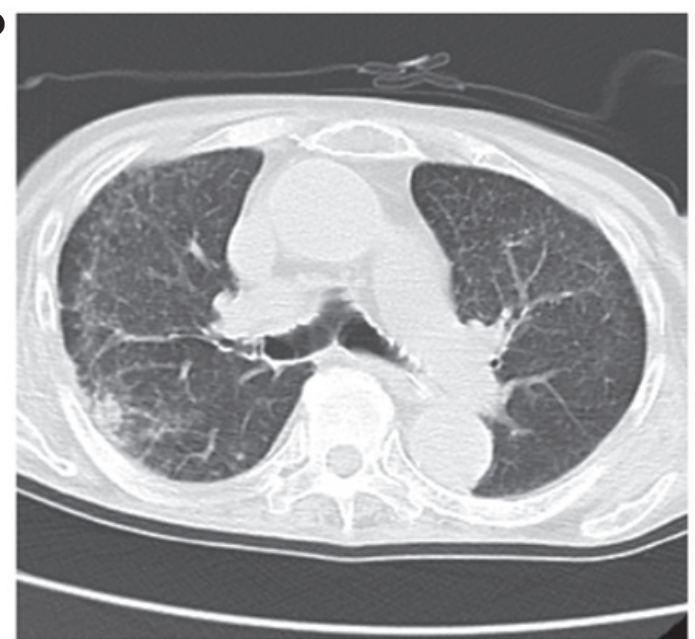

Figure 2. (A) Chest X-ray film and (B) CT scan presenting diffuse bilateral pulmonary infiltration. (C) Chest X-ray film presenting near absence of the diffuse bilateral consolidation observed previously, with a remaining patchy shadow in the right middle lung field. (D) CT scan presenting a diffuse, small nodular shadow and pale consolidation in the right $\mathrm{S} 2$ region. $\mathrm{CT}$, computerised tomography.

of the sputum, the patient was diagnosed with smear-negative, active pulmonary tuberculosis. Treatment was initiated with once daily $200 \mathrm{mg}$ isoniazid (Daiichi Sankyo Group, Tokyo, Japan), 300 mg rifampicin (Sandoz K.K., Tokyo, Japan) and 500 mg ethambutol (Kaken Pharmaceutical, Co., Ltd., Tokyo, Japan), and the patient was transferred to the Chronic Care Institution for the Aged.

Case 2. An 85-year-old woman was transported to Kainan Hospital by ambulance on 23rd March 2014 complaining of a high fever and chest tightness. In addition, the patient had been experiencing episodes of difficulty in swallowing for 1 month. The patient history included hypertension, atrial fibrillation, chronic kidney disease, pulmonary embolism and angina; these conditions were controlled by medication and an inferior vena cava filter. No clinical evidence of left atrial hypertension was detected by an ultrasonic cardiography performed on admission. The patient presented with the following signs: Temperature, $38.3^{\circ} \mathrm{C}$; pulse rate, 119 beats/min; blood pressure, $86 / 54 \mathrm{mmHg}$; and coarse crackles over the bilateral lungs. Edema in the legs was not detected on physical examination. Blood tests reported an increased percentage of neutrophils (white blood cells, $7,800 / \mathrm{mm}^{3} ; 94.2 \%$ neutrophils) and elevated levels of creatinine [2.47 mg/dl (normal range, $0.4-0.7 \mathrm{mg} / \mathrm{dl}$ )], urea nitrogen [75.3 mg/dl (normal range, 8.0-22.0 mg/dl)], lactate dehydrogenase [510 IU/1 (normal range, 119-229 IU/l)] and C-reactive protein $(12.09 \mathrm{mg} / \mathrm{dl})$. In addition, the patient had low total protein [5.8 g/dl (normal range, 6.7-8.3 IU/l)] and serum albumin $(2.4 \mathrm{~g} / \mathrm{dl})$ levels. A blood gas analysis revealed $\mathrm{pH}$ 7.467, $23.5 \mathrm{mmHg} \mathrm{PaCO}_{2}, 107 \mathrm{mmHg} \mathrm{PaO}_{2}$ and $99 \% \mathrm{SaO}_{2}$ with oxygen inhalation through a mask with a reservoir bag $(6 \mathrm{l} / \mathrm{min})$. The $\mathrm{PaO}_{2}: \mathrm{FiO}_{2}$ ratio was 178 . Chest X-ray film and $\mathrm{CT}$ identified diffuse bilateral pulmonary infiltrates (Fig. 2A and B). The sputum test for acid-fast bacillus at the time of admission was smear negative.

Based on the results of the examination and the episodes of difficulty in swallowing, the patient was clinically diagnosed with ARDS resulting from aspiration pneumonia. Meropenem ( 0.5 g every $12 \mathrm{~h}$; Meiji Seika Pharma,Co.,Ltd.) and minocycline (100 mg once daily; Sawai Pharmaceutical, Co., Ltd., Tokyo, Japan) antibiotics were administered for 11 days, in addition to oxygen therapy and intravenous feeding. The treatment was successful (improved oxygenation and a reduced fever) and the patient was discharged to a nursing home on 6th April. A chest X-ray film obtained at the time of discharge from the hospital revealed that the diffuse bilateral consolidation had almost 
disappeared; however, a patchy shadow remained in the right middle lung field (Fig. 2C). On 14th April, an acid-fast bacillus examination of the sputum obtained from the patient upon admission revealed culture-positive results, and a PCR test for M.tuberculosis was positive. The patient was immediately readmitted to the hospital and underwent sputum tests and a chest CT scan. The chest CT scan identified diffuse bilateral micro-nodules and pale consolidation in the right $\mathrm{S} 2$ region; these nodules and consolidation were proposed to be the focus of the tuberculosis infection (Fig. 2D). In addition, acid-fast bacillus re-examination of the sputum revealed smear-positive results. The patient was diagnosed with smear-positive, active pulmonary tuberculosis and was transferred to the National Hospital Organization Higashinagoya National Hospital (Nagoya, Japan) for anti-tuberculous treatment with isoniazid (200 mg once daily), rifampicin (300 mg once daily) and ethambutol (500 mg once daily). Fortunately, nosocomial transmission of tuberculosis from this patient to other patients and/or medical staff was not reported.

\section{Discussion}

The present study reports two cases of aspiration pneumonia with bilateral pulmonary infiltrates that resulted in the delayed diagnosis of pulmonary tuberculosis. The patients were diagnosed with pulmonary tuberculosis following discharge from the hospital, possibly due to the fact that the acid-fast bacillus examination of sputum was performed only once and the result was smear-negative/culture-positive. Although it may be difficult to diagnose complicated pulmonary tuberculosis in all patients with aspiration pneumonia at the time of admission, clinicians should pay careful attention to the course of pulmonary infiltration and should perform the acid-fast bacillus test on the sputum a minimum of three times.

The increasing ratio of elderly patients with newly diagnosed tuberculosis is a major medical and healthcare issue in Japan $(6,7)$. The incidence of tuberculosis infection was frequent in Japan prior to the 1960s, and the aging of these infected individuals is one of the reasons for the relatively high prevalence in Japan at present $(7-9,11)$. Elderly patients tend not to present with typical symptoms, such as weight loss, coughing and fever, and may not be able to expectorate sputum spontaneously, resulting in a delayed diagnosis $(6,7,9,12,13)$. In addition, as the life expectancy of the Japanese population increases, the increasing incidence of aspiration pneumonia in elderly patients emerges as a key medical and healthcare concern $(1,2,4,5,14,15)$. As symptoms of aspiration pneumonia tend to be non-specific and overlap with those of pulmonary tuberculosis, the risk of aspiration pneumonia combined with pulmonary tuberculosis is prominent in medical care for elderly patients $(6,16)$.

The diagnostic delay of tuberculosis can be divided into patient and doctor delay $(12,16,17)$. Patient delay refers to the time from the onset of symptoms to the first visit to a medical institution, and doctor delay refers to the time from the patient's first visit to the doctor to diagnosis. In elderly individuals, patient delay is shorter than in younger individuals, and doctor delay affects a relatively high percentage of cases in the elderly population $(16,17)$. The major causes for late diagnosis following a visit to a medical institution can be divided into two types: i) The doctor did not suspect pulmonary tuberculosis at the first visit; or ii) the results of the acid-fast bacillus examination were delayed (18). When elderly patients with pulmonary tuberculosis visit a hospital or arrive there by ambulance, they are typically diagnosed with aspiration pneumonia (6). In addition, elderly patients are more frequently diagnosed with tuberculosis at the hospital while receiving treatment for other illnesses, in comparison with younger patients (17). These observations suggest that diagnosing active tuberculosis is difficult in elderly patients as a result of nonspecific symptoms that may be confused with other diseases or the aging process. To improve this issue, medical staff involved in the care of elderly patients should be on alert for tuberculosis infection and should perform adequate examinations, including sputum tests for acid-fast bacillus, at the first opportunity.

In the two cases presented in the current study, an age-associated decline in immune function was considered to have affected the development of tuberculosis and to have contributed to a decline in the patients' general conditions. In addition, the compromised general condition of these patients may have facilitated the development of aspiration pneumonia. Finally, resulting from a reduction in $\mathrm{SaO}_{2}$, they were transported to the hospital by ambulance and were initially diagnosed with ARDS caused by aspiration pneumonia. On evaluation of the chest CT scans at the time of readmission, tuberculous lesions, such as nodules with satellite lesions and centrilobular micronodules, were detected. However, at the time of first admission, these tuberculous lesions were obscured by the diffuse bilateral pulmonary infiltration associated with ARDS. In addition, the smear-negative result in the sputum test for acid-fast bacillus at the time of initial admission may have had a significant impact on the delay in diagnosis. Considering the cases presented in the current study, the following two strategies to prevent diagnostic delay of pulmonary tuberculosis in elderly aspiration pneumonia patients should be adopted: i) The sputum test for acid-fast bacillus examination should be performed at least three times upon initial admission to hospital; and ii) if the observations are equivocal on chest X-ray films, regardless of any improvement of aspiration pneumonia, a chest CT should be performed to closely evaluate the shadow on the film.

In summary, the present study describes two cases of pulmonary tuberculosis complicated with aspiration pneumonia. Early diagnosis of pulmonary tuberculosis was difficult in the present cases, and acid-fast bacillus examination of the sputum should have been performed more than once in order to prevent nosocomial spread of tuberculosis. These cases should remind physicians to consider the clinical significance of pulmonary tuberculosis, which may provide further insight into aspiration pneumonia in elderly patients.

\section{References}

1. Tomono K: Stop Pneumonia Campaign. Nihon Kyobu Rinsho 73: S238-S243, 2014 (In Japanese).

2. Ishida T: Epidemiology of pneumonia in Japan - current status and future considerations. Nihon Naika Gakkai Zasshi 100: 3484-3489, 2011 (In Japanese).

3. Ota K and Shibata S: Diagnosis and medical treatment of aspiration pneumonia. J Clin Rehab 22: 877-885, 2013 (In Japanese). 
4. Teramoto S, Fukuchi Y, Sasaki H, Sato K, Sekizawa K and Matsuse T; Japenese Study Group on Aspiration Pulmonary Disease: High incidence of aspiration pneumonia in community- and hospital-acquired pneumonia in hospitalized patients: A multicenter, prospective study in Japan. J Am Geriatr Soc 56: 577-579, 2008.

5. Komiya K, Ishii H, Umeki K, Mizunoe S, Okada F, Johkoh T and Kadota J: Impact of aspiration pneumonia in patients with community-acquired pneumonia and healthcare-associated pneumonia: A multicenter retrospective cohort study. Respirology 18: 514-521, 2013.

6. Ubukata S, Jingu D, Yajima T, Shoji M and Takahashi H: Occurrence and clinical characteristics of tuberculosis among home medical care patients. Kekkaku 89: 649-654, 2014 (In Japanese).

7. Toyota E, Machida K, Nagayama N, Yamane A, Komiya K, Ito S, Suzuki J, Kashizaki F, Shimada M, Matsui Y, et al: Clinical investigation among elderly patients with tuberculosis. Kekkaku 85: 655-660, 2010 (In Japanese).

8. Tuberculosis Surveillance Center; RIT; JATA: Tuberculosis Annual Report 2012. (1). Summary of tuberculosis notification statistics and foreign-born tuberculosis patients. Kekkaku 89: 619-625, 2014 (In Japanese).

9. Fukushima Y, Shiobara K, Shiobara T, Tatewaki M, Anzai M, Fukushima F, Yamada I, Hirata H, Sugiyama K and Fukuda T: Patients in whom active tuberculosis was diagnosed after admission to a Japanese university hospital from 2005 through 2007. J Infect Chemother 17: 652-657, 2011.

10. Bernard GR, Artigas A, Brigham KL, Carlet J, Falke K, Hudson L, Lamy M,Legall JR, Morris A and Spragg R: The American-European Consensus Conference on ARDS. Definitions, mechanisms, relevant outcomes, and clinical trial coordination. Am J Respir Crit Care Med 149: 818-824, 1994
11. Tuberculosis Surveillance Center; RIT; JATA: Tuberculosis annual report 2012 - (2) Childhood and elderly tuberculosis. Kekkaku 89: 673-678, 2014 (In Japanese).

12. Lin CY, Lin WR, Chen TC, Lu PL, Huang PM, Tsai ZR, Huang MS, Tsai WC and Chen YH: Why is in-hospital diagnosis of pulmonary tuberculosis delayed in southern Taiwan? J Formos Med Assoc 109: 269-277, 2010.

13. Rajagopalan S: Tuberculosis and aging: A global health problem. Clin Infect Dis 33: 1034-1039, 2001.

14. Kadota J: Guideline for nursing and healthcare-associated pneumonia (NHCAP). Nippon Naika Gakkai Zasshi 100: 3503-3509, 2011 (In Japanese).

15. Teramoto S: Preventive strategy for reccuurent aspiration pneumoina. Nippon Naika Gakkai Zasshi 100: 3578-3585, 2011 (In Japanese).

16. Akagawa S: Present situation and practical treatment of elderly tuberculosis patients in Japan. Nippon Ronen Igakkai Zasshi 47 165-173, 2010 (In Japanese).

17. Ohmori M, Ozasa K, Mori T, Wada M, Yoshiyama T, Aoki M, Uchimura $\mathrm{K}$ and Ishikawa N: Trends of delays in tuberculosis case finding in Japan and associated factors. Int $\mathbf{J}$ Tuberc Lung Dis 9: 999-1005, 2005

18. Yamamoto K, Osumi M, Kinoshita A, Matsuoka Y Yanagihara K, Sakito O, Inoue Y, Fukushima K, Yonekura M and Kohno S: Incidence of tuberculosis and evaluation of diagnostic delay in admitted patients during past four years at national hospital organization Nagasaki Medical Center. IRYO 62: 323-330, 2008 (In Japanese). 